\title{
EFECTO DE TRES ESPECIES DE LOMBRICES EN LA FERTILIDAD DEL SUELO Y EL CRECIMIENTO INICIAL DEL MAÍZ (ZEA MAYS)
}

\author{
Esperanza Huerta, Olivia Valier, Day Romero \& Aaron Jarquin \\ Manejo y Fertilidad de Suelos. El Colegio de la Frontera Sur. Km 15.5 Carr. Villahermosa-Reforma, \\ Rancheria Guineo $2^{\mathrm{a}}$ Secc. Villahermosa Tabasco, cp. 86280, México. E-mail: ehuerta@ecosur.mx, \\ espehl@yahoo.es
}

Huerta, E., O. Valier, D. Romero \& A. Jarquin. 2010. Efecto de tres especies de lombrices en la fertilidad del suelo y el crecimiento inicial del maíz (Zea mays). Acta Zoológica Mexicana (n.s.), Número Especial 2: 219-226.

RESUMEN. En el presente experimento se evaluó el efecto de tres especies de lombrices de tierra en la fertilidad del suelo y el crecimiento del maíz (Zea mays), planta de gran importancia alimenticia en la republica Mexicana. Un individuo de cada especie (Balanteodrilus pearsei, Lavellodrilus bonampakensis, especies nativas y Pontoscolex corethrurus, especie exótica) fue utilizado en el experimento de germinación y crecimiento inicial del maíz (fase vegetativa v6), con cuatro repeticiones por tratamiento y 9 semillas por recipiente, con $4 \mathrm{~kg}$ de suelo cada uno. Se determinaron los contenidos de materia orgánica, nitrógeno total y fósforo disponible en el suelo y en las hojas de maíz al final del experimento. $L$. bonampakensis aumentó significativamente la biomasa foliar y radicular, $B$. pearsei aumentó solamente la biomasa aérea y $P$. corethrurus disminuyó la biomasa de raíces en relación al testigo. El contenido de materia orgánica en el suelo fue significativamente mayor con lombrices que sin lombrices. Para los demás parámetros no se observaron diferencias significativas entre los tratamientos con y sin lombrices. La presencia de lombrices puede afectar la fertilidad del suelo y el crecimiento inicial del maíz de forma positiva, negativa o neutra, dependiendo de la especie. Sin embargo, más estudios son necesarios para comprender mejor la relación de las especies nativas y exóticas de lombrices tropicales con las plantas y el suelo.

Palabras clave: Lombrices, fertilidad del suelo, crecimiento vegetal.

Huerta, E., O. Valier, D. Romero \& A. Jarquin. 2010. Effect off three earthworm species on soil fertility and the growth of maize (Zea mays) seedlings. Acta Zoológica Mexicana (n.s.), Número Especial 2: 219-226.

ABSTRACT. In the present experiment, we evaluated the effect of three earthworm species on soil fertility and seedling growth of maize (Zea mays), a food plant of great importance in Mexico. One individual of each species (Balanteodrilus pearsei and Lavellodrilus bonampakensis, two native species and Pontoscolex corethrurus, an exotic), were used in the germination and initial maize growth experiment (vegetative phase v6), with four replicates per treatment and 9 seeds per pot, with $4 \mathrm{~kg}$ of soil each. Organic matter contents, total nitrogen and available phosphorus in the soil and in the maize leaves

Recibido: 16/05/2008; aceptado: 08/01.2010. 
Huerta et al.: Lombrices, suelo y crecimiento inicial del maiz

were determined at the end of the experiment. L. bonampakensis significantly increased foliar and root biomass, $B$. pearsei increased only shoot biomass and $P$. corethrurus decreased root biomass in relation to the control. Soil organic matter content increased significantly with earthworms, but for the remaining parameters, no significant differences were found between treatments with and without earthworms. Earthworm presence can affect soil fertility and initial maize growth positively, negatively or not at all, depending on the species involved. However, further research is necessary to better understand the relationships between native and exotic tropical earthworm species, plants and the soil.

Key words: Earthworms, soil fertility, plant growth.

\section{INTRODUCCIÓN}

Para disminuir el uso de agroquímicos y la dependencia de la agricultura en fertilizantes inorgánicos, es necesario buscar métodos alternativos de manejo de la fertilidad de los suelos. Una de ellas es el manejo biológico del suelo (Swift 1999), incluyendo la inoculación de lombrices de tierra para restaurar las condiciones de suelos dañados o degradados y estimular la productividad agrícola (Butt et al. 1997, Brown et al. 1999, Lavelle et al. 1998). La actividad de las lombrices favorece el crecimiento de las plantas (Edwards \& Lofty 1980), y una biomasa mínima de $30 \mathrm{~g} \mathrm{~m}^{-2}$ ha producido efectos en la producción agrícola (Brown et al. 1999). Sin embargo, la inoculación de lombrices no es sencilla y se requieren de condiciones idóneas (cobertura arbórea, uso de leguminosas) para que tenga éxito. También faltan muchos datos sobre la relación de las lombrices con las plantas en el trópico; solamente algunas especies de lombrices y plantas, principalmente exóticas o peregrinas han sido estudiadas a la fecha. El objetivo de este trabajo fue el observar si existen diferencias en el contenido de nutrientes en el suelo y en las hojas de maíz en su fase vegetativa inicial al inocular tres especies de lombrices. Se utilizaron dos especies nativas de México, Balanteodrilus pearsei (Pickford 1938) y Lavellodrilus bonampakensis (Fragoso 1991) y una exótica, Pontoscolex corethrurus (Müller 1857) que ha sido encontrada de manera natural en sistemas agrícolas de Tabasco.

\section{MATERIAL Y MÉTODOS}

En las instalaciones del Colegio de la Frontera Sur ubicado en el km 15.5 de la carretera Villahermosa-Reforma, Tabasco, México, se llevo a cabo el experimento. Se colectaron lombrices juveniles de las especies L. bonampakensis, B. pearsei y $P$. corethrurus, que fueron cultivadas en condiciones estables de humedad (33\%) y temperatura $\left(27^{\circ} \mathrm{C}\right)$ en suelo enriquecido con $1.5 \%$ de Mucuna pruriens var. utilis de acuerdo al manual de Huerta (2008). Después de dos meses de crecimiento se obtuvieron individuos de $1.2 \pm 0.43 \mathrm{~g}$ los cuales fueron colocados uno por recipiente siguiendo la proporción de $30 \mathrm{~g} \mathrm{~m}^{-2}$ con el fin de observar efectos en la germinación y crecimiento del maíz (Zea mays). Los recipientes contaron con un tamaño de $20 \mathrm{~cm}$ de altura $\times 6 \mathrm{~cm}$ de diámetro y $4 \mathrm{~kg}$ de capacidad, con dos orificios pequeños en cada extremo con el fin de hacer drenar el recipiente. 
El suelo, clasificado como un fluvisol (de acuerdo a Palma \& Sánchez 2002) fue colectado y tamizado a $0.5 \mathrm{~mm}$. El suelo usado tenía un contenido de $4.5 \%$ de materia orgánica. En cada recipiente se sembraron 9 semillas de maíz. Cada tratamiento tuvo 4 repeticiones para un total de 16 recipientes. Una vez germinadas las plantas se dejaron únicamente 3 plantas por recipiente. Se monitoreo el tamaño de las plantas cada tercer día, inmediatamente después de la germinación, midiendo la altura con una cinta métrica por el tallo. Al cabo de 32 días después de la germinación, cuando las plantas presentaron de 6-8 hojas (fase vegetativa v6) se evaluó la biomasa de raíces y aérea del maíz cortando y pesando directamente. Se determinó el contenido de materia orgánica (MO) (Walkley \& Black 1934) y fósforo (P) disponible (Olsen 1982) en el suelo, se analizó el nitrógeno (N) y P total en las hojas de las plantas siguiendo la norma oficial mexicana (SEMARNAT 2000), y se cuantificaron y pesaron las lombrices en cada recipiente.

Se efectuaron análisis de varianzas para datos no paramétricos (por no tener una distribución normal), usando el Mann-Whitney U-test, con el fin de observar diferencias significativas entre los tratamientos. También se realizo un análisis de componentes principales usando 10 variables (contenido de $\mathrm{MO}$ y $\mathrm{P}$ en el suelo, contenido de $\mathrm{N}$ y $\mathrm{P}$ en hojas de maíz, altura de la planta, biomasa aérea por planta y por recipiente, $\%$ de germinación, biomasa de raíces por planta y por recipiente), con el fin de observar la relación entre las variables y como las diferentes especies de lombrices influyeron o no sobre dichas variables, con el programa ADE-4 (Thioulouse et al. 1997). De igual forma se hizo un test de Montecarlo con el fin de observar el grado de significancia del tipo del efecto de las especies sobre las diferentes variables testadas.

\section{RESULTADOS}

Del total de semillas colocadas por recipiente germinaron el $80 \%$ con la presencia de L. bonampakensis y $69 \%$ con $P$. corethrurus, pero no hubo diferencias significativas entre los tratamientos (Cuadro I). La altura de las plantas alcanzo un promedio de 70 $\mathrm{cm}$ en presencia de $L$. bonampakensis y $62 \mathrm{~cm}$ con $B$. pearsei, pero también no se observaron diferencias significativas entre los tratamientos (Cuadro I).

Las plantas con el mayor peso fresco $(68 \pm 14 \mathrm{~g})$ después de 32 días de crecimiento fueron las que crecieron en los recipientes con L. bonampakensis y B. pearsei, con valores significativamente mayores que aquellas en presencia de $P$. corethrurus y $\sin$ lombrices (Cuadro I). En cuanto al peso fresco de las raíces este fue significativamente mayor con inoculación de L. bonampakensis $(20.4 \pm 3 \mathrm{~g})$ en comparación a los demás tratamientos. En presencia de $P$. corethrurus, la biomasa de raíces fue menor que en el tratamiento testigo sin lombrices (Cuadro I).

El contenido de materia orgánica en el suelo fue significativamente mayor en presencia de las tres especies de lombrices que en el suelo sin lombrices (Cuadro I). 


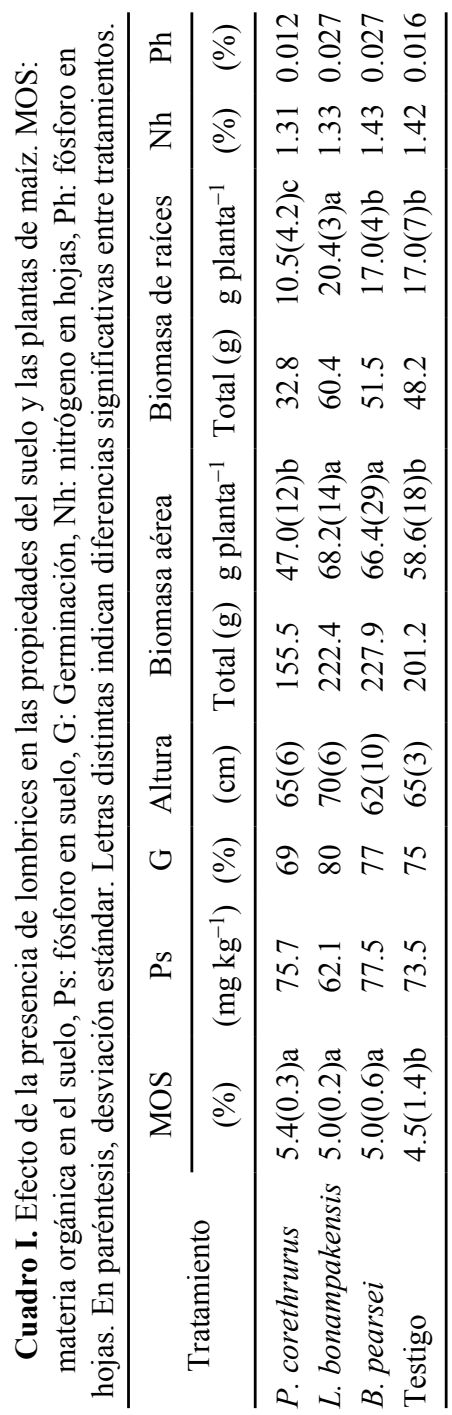


Para los demás parámetros, o sea $\mathrm{P}$ en el suelo, $\mathrm{N}$ y $\mathrm{P}$ en las hojas, no se observaron diferencias significativas entre los tratamientos.

El circulo de correlación del análisis de componentes principales, con $90 \%$ de inercia en ambos ejes (Fig. 1a), mostró que la altura del maíz se separó completamente de las otras variables, encontrándose en oposición al contenido de $\mathrm{P}$ en el suelo y $\mathrm{N}$ en las hojas. En el eje 1, el \% de germinación, la biomasa de raíces por planta y por recipiente estuvieron muy correlacionadas con el \% de fósforo en hojas y la biomasa aérea foliar por planta y recipiente. Al disponer las especies de lombrices sobre el plano factorial (Fig. 1b p: 0.0) se observó que $P$. corethrurus estuvo más correlacionado con altos contenidos de materia orgánica en el suelo, que $L$. bonampakensis produce efectos importantes en la altura del maíz y que $B$. pearsei promueve mayores contenidos de nitrógeno en las hojas de las plantas.

Las lombrices no presentaron un aumento significativo en su peso y no hubo reproducción de las mismas durante el experimento.

\section{DISCUSIÓN}

Las tres lombrices utilizadas pertenecen a la misma categoría ecológica (endogeas, Fragoso 2001) pero sus efectos sobre el suelo y las plantas de maíz fueron distintos: $P$. corethrurus tuvo mayores efectos sobre el contenido de materia orgánica en el
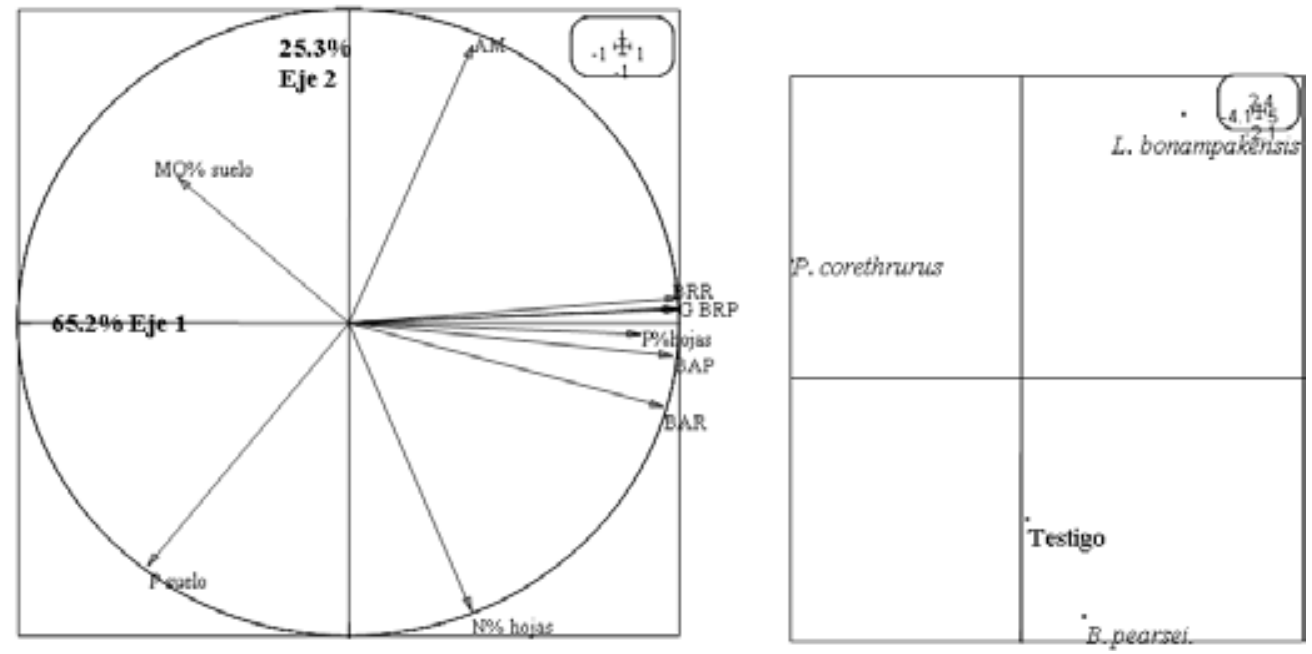

Figura 1. a) Circulo de correlación después de un análisis en componentes principales, matriz de correlación. $\mathrm{MO} \%$ : materia orgánica, $\mathrm{N}$ : nitrógeno, $\mathrm{P}$ : fósforo, $\mathrm{AM}$ : altura del maíz en el día 32, $\mathrm{BRR}$ : biomasa raíces del maíz por recipiente, BRP: Biomasa raíces del maíz por planta, BAR: biomasa aérea del maíz por recipiente, BAP: Biomasa aérea del maíz por planta, G: \% de germinación; b) Especies de lombrices en el plano factorial después de un análisis en componentes principales (p:0.00). 
suelo; B. pearsei sobre los contenidos de nitrógeno en las hojas, L. bonampakensis, $B$. pearsei (positivos) y P. corethrurus (negativo) sobre la biomasa aérea y de raíces, respectivamente. L. bonampakensis promovió un incremento del $30 \%$ de la biomasa aérea en comparación con las plantas en presencia de $P$. corethrurus, pero este valor sólo fue $12.7 \%$ mayor que el testigo. Esta es la primera vez que se reportan los efectos de estas especies de lombrices sobre el crecimiento inicial del maíz. Otros autores, han estudiado principalmente el efecto de las lombrices sobre la producción de maíz y su biomasa en la época de la cosecha de los granos. En esos estudios, Ortiz et al. (2007) no encontraron efectos de $B$. pearsei sobre la producción de maíz y Brown et al. (2004a) observaron efectos negativos y neutros de $P$. corethrurus sobre la biomasa de maíz en presencia y ausencia de residuos de maíz, respectivamente. En el Perú, Pashanasi et al. (1994) observó un incremento del 37\% de la producción de maíz en presencia de $P$. corethrurus y leguminosas.

Scheu (2003) y Brown et al. (2004b) mencionan que alrededor de un 9\% de los trabajos que investigan la relación entre las lombrices y las plantas muestran efectos negativos de las lombrices a las plantas, aunque el efecto depende del sistema, los inputs suministrados y el $\mathrm{pH}$, textura y \%C del suelo (Ortiz et al. 2007). El presente estudio se llevo a cabo con el fin de observar cual lombriz produce los mejores efectos sobre las plantas de maíz y el suelo y después poder recomendar su reproducción al agricultor. Los contenidos de fósforo reportados en las hojas del maíz corresponden a rangos medios (0.03-0.5\%) de suficiencia evaluados por Jones et al. (1991), adecuados para que las plantas puedan crecer sin uso de fertilizante fosforado, aunque esto depende de la edad de la planta; a mayor edad, mayor es el requerimiento.

Hasta la fecha solo se han manejado solamente el $8 \%$ de las especies de lombrices de la republica mexicana (Fragoso 2001), y seria conveniente el promover su reproducción y uso pero es necesario comprender con mayor exactitud la relación de las mismas con las plantas y los suelos. Algunos puntos importantes para considerarse en esta relación son: 1) la categoría ecológica de la lombriz, lo que influirá en su alimentación y en la cantidad de suelo, tipo y calidad de recursos orgánicos que las lombrices utilicen en el sistema; 2) la relación simbiótica que presentan con su microflora (Barois 1987), lo que hace o hará que las lombrices tengan mayor o menor éxito en utilizar los nutrientes del suelo y los efectos que produzcan sobre las plantas; 3) si son nativas o exóticas, pues las lombrices nativas, al ser menos agresivas en ocupar y usar recursos de los nuevos ambientes podrían promover mayores beneficios a las plantas. Más estudios son necesarios con el fin de comprender la relación de las lombrices nativas y exóticas tropicales con las plantas.

\section{AGRADECIMIENTOS}

Al Dr. Regino Gómez por sus consejos. Al proyecto TAB-2007-C09-74864. A fondos mixtos Tabasco proyecto 11316 quien financio el presente estudio y a los reviso- 
res de este documento por sus observaciones muy acertadas y a Traducciones Glazer quien hizo la traducción del resumen.

\section{LITERATURA CITADA}

Barois, I. 1987. Interactions entre les vers de terre géophages et la microflore du sol pour l'exploitation de la matière organique du sol. Travaux de Recherche de Lamto. 7: 1-151.

Brown, G., B. Pashanasi, C. Villenave, J. Patron, B. Senapati, S. Giri, I. Barois, P. Lavelle, E. Blanchart, R. Blakemore, A. Spain \& J. Boyer. 1999. Effects of earthworms on plant production in tropics. Pp. 87-147. In: P. Lavelle, L. Brussaard and P. F. Hendrix (Eds.). Earthworm management in tropical agroecosystems. CAB-International Wallingford.

Brown, G. G., J. C. Patrón, I. Barois \& P. Lavelle. 2004a. Tropical earthworm (Pontoscolex corethrurus: Glossoscolecidae; Polypheretima elongata: Megascolecidae) effects on common bean (Phaseolus vulgaris) and maize (Zea mays) production under greenhouse conditions. Pp. 313-339. In: S. H. S. Hanna and W. Z. A. Mikhail (Eds.). Soil zoology for sustainable development in the 21st century. Palm Press, Cairo.

Brown, G. G., C. A. Edwards \& L. Brussaard. 2004b. How earthworms affect plant growth: burrowing into the mechanisms. Pp. 13-49. In: C. A. Edwards (Ed.). Earthworm ecology. CRC Press, Boca Raton.

Butt, K. R., J. Frederickson \& R. M. Morris. 1997. The Earthworm Inoculation Unit (EIU) technique, an integrated system for cultivation and soil-inoculation of earthworms. Soil Biology \& Biochemistry. 29(3/4): 251-257.

Edwards, C. \& J. R. Lofty. 1980. Effects of earthworm inoculation upon the root growth of direct drilled cereals. Journal of Applied Ecology, 17(3): 533-543.

Fragoso, C. 2001. Las lombrices de tierra de México (Annelida, Oligochaeta): diversidad, ecología y manejo. Acta Zoológica Mexicana (nueva serie). Numero especial 1: 131-171

Fragoso, C. 1991. Two new species of earthworm genus Lavellodrilus (Oligochaeta, Acanthodrilini) from tropical Mexican rain forests. Studies on Neotropical Fauna and Environment. 26(2): 83-91.

Huerta, E. 2008. Manual de reproducción de lombrices nativas. El Colegio de la Frontera Sur. México.

Jones, B. J., B. Wolf \& H. A. Mills. 1991. Plant analysis handbook: a practical sampling, preparation, analysis and interpretation guide. Micro-Macro publishing, Athens.

Lavelle, P., I. Barois, E. Blanchart, G. Brown, T. Decaëns, C. Fragoso, J. J. Jimenez, K. Kajondo, M. Martinez, A. Moreno, B. Pashanasi, B. Senapati \& C. Villenave. 1998. Earthworms as a resource in tropical agroecosystems. Nature \& Resources. 34(1): 26-41.

Olsen, S. R. \& L. E. Sommers. 1982. Phosphorus. Pp 57-62. In: A. L. Page, R. H. Miller and D. R. Keeney (Eds). Methods of soil analysis, Part 2, $2^{\text {nd }}$ Ed. American Society of Agronomy, Madison.

Ortiz-Ceballos A. I., C. Fragoso \& G. G. Brown. 2007. Synergistic effect of a tropical earthworm Balanteodrilus pearsei and velvetbean Mucuna pruriens var. utilis on maize growth and crop production. Applied Soil Ecology. 35: 356-362.

Palma-Lopez, D. J. \& A. Triano-Sanchez. 2002. Plan de uso sustentable de suelos de Tabasco II. Colegio de Postgraduados, Cárdenas.

Pashanasi, B., P. Lavelle \& J. Alegre. 1994. Efecto de lombrices de tierra (Pontoscolex corethrurus) sobre el crecimiento de cultivos anuales y características físicas y químicas en suelos de Yurimaguas. Folia Amazónica 6: 5-45.

Pickford, E. 1938. Earthworms in Yucatán Caves. Carnegie Institution of Washington. 491: 71-100. 
Huerta et al.: Lombrices, suelo y crecimiento inicial del maiz

Secretaria del Medio Ambiente y Recursos Naturales. 2000. Norma Oficial Mexicana NOM-021RECNAT-2000. SEMARNAT, México.

Scheu, S. 2003. Effects of earthworms on plant growth: patterns and perspectives. Pedobiologia 47: 846-856.

Swift, M. J. 1999. Towards the second paradigm: Integrated biological management of soil. Pp. 11-24. In: J. O. Siqueira, F. M. S. Moreira, A. S. Lopes, L. R. G. Guilherme, V. Faquin, Neto Furtani, A. E. and J. G. Carvalho. (Eds). Inter-relação fertilidade, biologia do solo e nutrição de plantas. UFLA, Lavras.

Thioulouse, J., D. Chessel, S. Dolédec \& J-P. Olivier. 1997. ADE-4: a multivariate analysis and graphical display software. Statistics and Computing. 7: 75-83.

Walkley, A. \& L. A. Black. 1934. An examination of Degtjareff method for determining soil organic matter and proposed modification of the chromic acid titration method. Soil Science. 37: 29-38. 